\title{
Harmony Triggering as a Contrastive Property of Segments
}

\author{
Caitlin Smith \\ University of Southern California
}

\section{Introduction}

A harmony process is characterized by the spreading of a feature from a trigger segment onto one or more undergoer segments. In some languages, any segment bearing a harmonizing feature will trigger harmony. In other languages, some segments bearing a harmonizing feature trigger harmony while other segments bearing that feature do not. This paper examines such a case in Classical Manchu tongue root harmony, in which some advanced tongue root (ATR) vowels idiosyncratically trigger a process of ATR harmony while others do not.

I propose that this pattern and others like it are best accounted for by an analysis in which a segment's status as a trigger of harmony is encoded within its subsegmental representation, and a phonological grammar shapes a language's inventory to include both harmony-triggering and non-triggering segments. This analysis is implemented within the Gestural Harmony Model proposed by (Smith 2016a, 2016b), in which the assumed units of phonological representation are dynamically-defined gestures, following Browman \& Goldstein $(1986,1989)$. A common alternative approach to accounting for phonological exceptionality, indexation between morphemes and constraints (Pater 2000, 2009), cannot be successfully applied to exceptional triggering of harmony. Such indexation to harmony-driving constraints makes several undesirable predictions, both over- and under-generating patterns of exceptional triggering.

The rest of the paper is organized as follows. Section 2 lays out the basic pattern of ATR harmony in Classical Manchu. Section 3 reviews the workings of the Gestural Harmony Model introduced by Smith (2016a, 2016b), while section 4 presents an analysis of Classical Manchu ATR harmony within that model. Section 5 describes the undesirable predictions made by analyses that rely on constraint indexation to harmony driving constraints to account for patterns of apparent exceptionality in harmony triggering. Section 6 concludes.

\section{Tongue Root Harmony in Classical Manchu}

As in many Tungusic languages, harmony processes determine patterns of vowel co-occurrence in Classical Manchu. ${ }^{1}$ While early sources cited by Vago (1973) treat the harmony system of Classical Manchu as one based on vowel backness, Zhang (1996) argues that the assignment of vowels into one of two harmony classes is best understood based on tongue root position. According to Zhang, Classical Manchu has the inventory depicted in (1), with vowels divided into ATR and RTR (retracted tongue root) harmony classes.

\footnotetext{
* Thanks to the members of USC's Phonlunch group, as well as audiences at the Southern California April Meeting on Phonology (SCAMP), AMP 2016, and NELS 47. I would especially like to thank Rachel Walker, Karen Jesney, Louis Goldstein, Charlie O'Hara, Hayeun Jang, and Brian Smith for their comments on this work.

${ }^{1}$ In addition to the tongue root harmony discussed in this paper, Classical Manchu also displays a rounding harmony pattern that is only triggered when the nonhigh vowel / $/$ / occurs in the first two syllables of the word. While interesting, a full discussion of this pattern is outside the scope of this paper. See Zhang (1996), Zhang \& Dresher (1996), and Walker (2001) for discussion of Classical Manchu rounding harmony.
} 


\begin{tabular}{ccc}
\hline \multicolumn{2}{c}{ ATR Vowels } & RTR Vowels \\
\hline $\mathrm{i}$ & $\mathrm{u}$ & $\mathrm{U}$ \\
& $\partial$ & $\mathrm{a} \rho$ \\
\hline
\end{tabular}

In the tongue root harmony system of Classical Manchu, RTR / / alternates with ATR /u/, and RTR /a/ alternates with $/ \mathrm{\partial} /$ for tongue root position and with $/ \mathrm{\partial} /$ for rounding. ATR harmony may be triggered when an ATR vowel occurs in an initial syllable. The nonhigh ATR vowel /a/ always triggers harmony when it occurs in an initial syllable, as in (2a-c). As a result, all vowels that follow must be ATR as well. This holds both within roots and between roots and suffixes, most of which have ATR and RTR alternants. Nonhigh ATR /a/ may never occur in a non-initial syllable except as a product of harmony, i.e., following a harmony-triggering ATR vowel (2d-f). (All data are from Zhang (1996).)
a. [səbృə-ngə] 'joyous'
d. [kimu-nga] 'harboring hatred'
b. [hərə-ku] 'ladle'
e. [sisə-ku] 'sieve'
c. [hatu-kən] 'somewhat stocky'
f. [uјə-kən] 'somewhat heavy'

If the RTR vowels /a/ or / $/$ occur in an initial syllable, a following nonhigh vowel must surface as RTR /a/, as in (3). High vowels in non-initial syllables are unrestricted in terms of tongue root specification, with $/ \mathrm{i} /$, $/ \mathrm{u} /$, and $/ \mathrm{v} /$ all occurring in those positions $(3 \mathrm{c}-\mathrm{f}){ }^{2}$ When $/ \mathrm{i} / \mathrm{and} / \mathrm{u} /$ occur non-initially they never trigger ATR harmony in following vowels (3d-f).
a. [aga-nga] 'of rain'
b. [hola-nga] 'crying'
c. [malho-nga] 'frugal'
d. [kani-nga] 'agreeing'
e. [goni-nga] 'thoughtful'
f. [hodu-kan] 'somewhat fast'

Of particular interest here is the fact that the high ATR vowels [i] and [u] do not uniformly trigger ATR harmony when they occur in an initial syllable, as is the case for non-high [ə]. Instead, harmony is triggered in some lexical items and not in others. In (4a-e), [i] and [u] trigger ATR harmony within and across morpheme boundaries, while in (4f-i) ATR harmony is not triggered.
a. [sito-ku] 'bed-wetter'
f. [nimasa-kv] 'two-man boat'
b. [cisu-də] 'act for interest'
g. [nilho-da] 'be slick'
c. [hurə-nə] 'arch'
d. [urgu-nge] 'joyous'
h. [tuwa-na] 'go to look'
i. [muko-ha-] 'held a liquid in the mouth'

e. [ungi-ha] 'sent off'

An analysis of this complex pattern of triggering in Classical Manchu is made possible by adopting a gestural representation of harmony. The following section reviews how the triggering and undergoing of harmony are represented in gestural phonology before turning to an analysis of the specific pattern of triggering in Classical Manchu.

\section{Triggering in the Gestural Harmony Model}

In the Gestural Harmony Model outlined by Smith (2016a, 2016b), harmony is the result of the overlap of one or more target gestures by a triggering gesture of prolonged duration. The gestural units that are adopted within this model are modified from those defined within the framework of Articulatory

\footnotetext{
${ }^{2}$ The high front vowel does not have an RTR counterpart and always surfaces as ATR [i]. I assume that this is due to an undominated markedness constraint prohibiting [I], though this constraint is not included in the analysis that follows.
} 
Phonology (Browman \& Goldstein 1986, 1989). Gestures are phonological units that are specified for some goal articulatory state, such as 'closed lips' or 'advanced tongue root.' In addition, each gesture is specified for a number of parameters that determine how that goal state is reached, including which articulators to move, how strongly the gesture can command those articulators, and how quickly the goal state is attained. The Gestural Harmony Model also references a parameter that specifies whether a gesture self-deactivates when its goal state is reached. This is crucial to representing whether a gesture is a trigger of harmony.

The figure in (5) compares two tongue root advancement gestures that differ only in the setting of this deactivation parameter. The top gesture self-deactivates once its goal articulatory state, an advanced tongue root, is achieved; this is indicated by the stop sign icon. Following this deactivation, the tongue root returns to its neutral position. In contrast, the bottom gesture achieves its goal state and remains active rather than self-deactivating. The resulting prolonged duration of this gesture, a harmony trigger, results in its overlap with any following gestures, the undergoers of harmony. (The horizontal lengths of the boxes indicate gestural durations; the gradually climbing and falling lines represent degree of tongue root advancement.)

$$
\text { Self-deactivating and non-self-deactivating tongue root advancement gestures }
$$
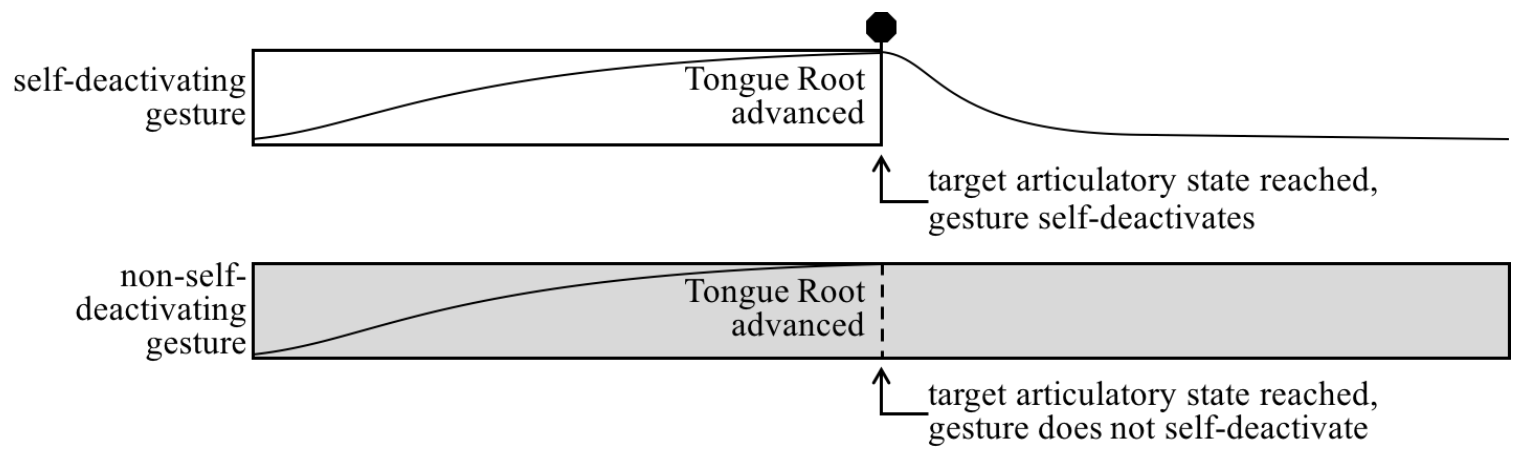

In the Gestural Harmony Model, harmony is not directly motivated by the satisfaction of a harmonydriving constraint. Rather, a segment will trigger harmony if one of its composite gestures surfaces as nonself-deactivating. Patterns of harmony triggering are the result of shaping of a language's phonological inventory, and therefore its surface forms, to contain either self-deactivating or non-self-deactivating gestures. This inventory shaping is conducted via the interaction of markedness and faithfulness constraints, the most important of which are described here.

Most gestures are self-deactivating and therefore do not trigger harmony. This is in accordance with the constraint SELFDEACTIVATE, which penalizes non-self-deactivating gestures.

SelfDeACTIVATE: Assign a violation mark to a gesture that is non-self-deactivating.

However, certain types of gestures do trigger harmony in some languages; these include lip protrusion gestures for rounding harmony and tongue root advancement gestures for ATR harmony. Such gestures trigger harmony in order to satisfy the constraint NONSELFDeACTIVATE $\left(\mathrm{Gest}_{\mathrm{X}}\right)$, which penalizes gestures of certain types that surface as self-deactivating.

NonSelfDeactivate(Gest $\mathrm{X})$ : Assign a violation mark to a gesture of type $\mathrm{X}$ that is selfdeactivating.

Both of these markedness constraint motivate the potential alteration of a gesture's underlying deactivation parameter setting. This underlying setting is preserved by the faithfulness constraint IDENT(deactivation). When SelfDeactivate is ranked above Ident(deactivation) and NonSelfDeactivate(Gest ${ }_{\mathrm{X}}$ ), the result is a language with no harmony in which all gestures surface as typical self-deactivating gestures. However, when NonSelfDeactivate(Gest $\mathrm{X}$ ) is ranked above Ident(deactivation) and SelfDeactivate, gestures of type $\mathrm{X}$ in that language will always trigger harmony, whether they are underlyingly specified to 
potentially extend in duration or to self-deactivate. All possible input gestures of type $\mathrm{X}$ will neutralize to the non-self-deactivating type.

The representation of a phonological unit's ability to trigger harmony as an encoded property of that unit, and therefore a property that can be preserved by a faithfulness constraint, is a crucial innovation of the Gestural Harmony Model. It introduces the ability of a gesture's deactivation parameter to serve a contrastive function. When IDENT(deactivation) is ranked above both SELFDEACTIVATE and NONSELFDeActivate(Gest X $_{\mathrm{X}}$, two gestures are capable of contrasting according to each's ability or inability to trigger harmony. The following section takes advantage of this to account for the idiosyncratic ability of high ATR vowels to trigger harmony in Classical Manchu.

\section{Gestural Analysis of Classical Manchu ATR Harmony}

4.1 Harmony Triggering in the Phonological Inventory Now that the basic workings of the Gestural Harmony Model have been introduced, this section presents an analysis of the complex pattern of harmony triggering in Classical Manchu. Recall from section 2 that in this language triggers of progressive ATR harmony are restricted to the initial syllable of a root. In this position, nonhigh /a/ always triggers harmony while /i/ and /u/ serve as triggers in some lexical items and not others. This type of pattern is best analyzed within a framework in which a segment's status as a trigger or non-trigger of harmony is directly encoded in the representation of that segment, making it able to serve a contrastive function. I propose that the seemingly inconsistent ability of /i/ and / $\mathrm{u} /$ to trigger ATR harmony can be explained by positing that the phonological inventory of Classical Manchu actually contains two /i/ vowels and two /u/ vowels that contrast in their ability or inability to trigger harmony.

Such an analysis is made possible by adopting the Gestural Harmony Model, in which a gesture's ability to trigger harmony is encoded by the setting of its self-deactivation parameter. As discussed in section 3, a language will display tongue root harmony if one or more of the vowels in its inventory includes a non-self-deactivating tongue root gesture. For each pair of high ATR vowels, one is specified as a trigger, accompanied by a non-self-deactivating tongue root advancement gesture, and one is specified as a non-trigger, accompanied by a self-deactivating tongue root advancement gesture. The nonhigh ATR vowel $/ \mathrm{a} /$, on the other hand, triggers harmony and must therefore be accompanied by a non-selfdeactivating tongue root advancement gesture. Taking all of this into account, the inventory of ATR vowels in Classical Manchu is represented gesturally as in (8):

Classical Manchu ATR Vowel Inventory

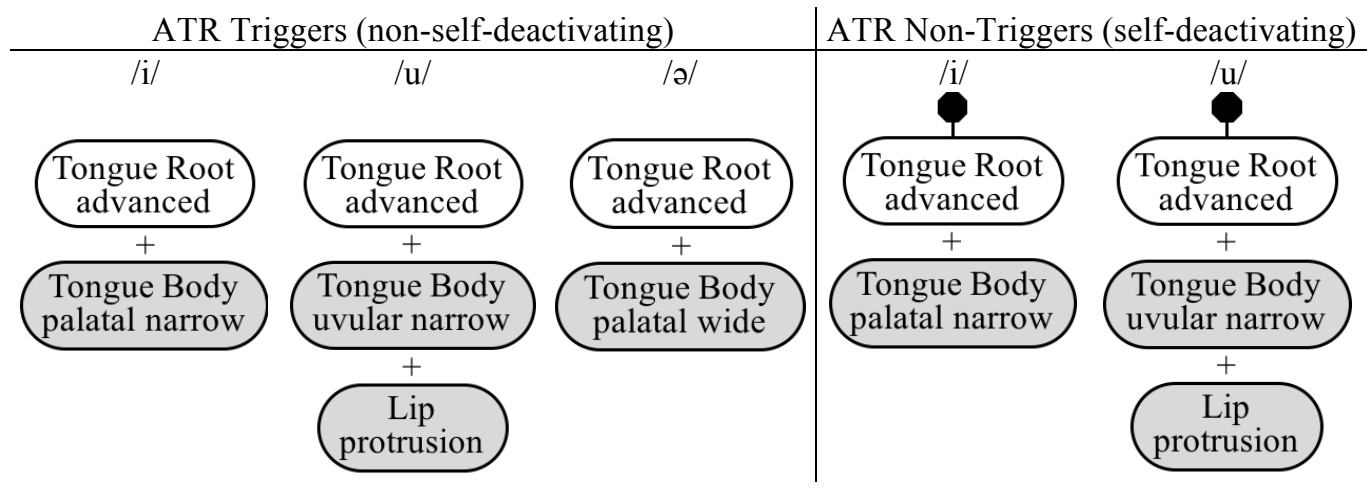

The vowels in the Classical Manchu inventory are represented in (8) as sets of one or more concurrently active gestures. An RTR vowel is represented by a single tongue body gesture, while its ATR counterpart is represented by a tongue body gesture accompanied by a tongue root advancement gesture. (A round vowel also includes a lip protrusion gesture.) Crucial to the analysis of Classical Manchu in the Gestural Harmony Model is the fact that this inventory contains self-deactivating and non-self-deactivating tongue root advancement gestures, which serves to divide this inventory into sets of triggers and non-triggers of ATR harmony. 
4.2 Harmony Triggering Patterns via Constraint Interaction In the Gestural Harmony Model, the pattern of harmony triggering in a language is determined by the shape of that language's phonological inventory and the distributions of the members of that inventory. Any analysis conducted in this framework must be based on the interaction of the markedness and faithfulness constraints introduced in section 3 . The remainder of this section outlines such an analysis for Classical Manchu tongue root harmony.

The first step in this analysis is determining the constraint ranking necessary to account for the distributions of the high ATR vowels. These vowels are contrastive with respect to their statuses as harmony triggers; however, this contrast is positionally sensitive. In non-initial syllables, a high ATR vowel may never trigger harmony and therefore must always surface with a self-deactivating tongue root advancement gesture. Therefore, the constraint SelfDeActivate must be ranked above IDENT(deactivation) and NONSELFDEACTIVATE(ATR), which calls for tongue root gestures to surface as non-self-deactivating. With the markedness constraint SELFDEACTIVATE ranked high, the grammar will rule out any tongue root advancement gestures that are not self-deactivating, no matter the underlying specification of a gesture's deactivation parameter.

In the privileged position of the initial syllable, however, this contrast is preserved. Both triggering and non-triggering $/ \mathrm{i} /$ and $/ \mathrm{u} /$ may occur in initial syllables, indicating that both non-self-deactivating and selfdeactivating tongue root advancement gestures may surface in that position. Beckman (1997, 1998) proposes that contrasts may be specially preserved in privileged positions via positional faithfulness constraints. Following this proposal, the contrast between triggering and non-triggering high ATR vowels in Classical Manchu can be achieved by positing a positional version of the constraint IDENT(deactivation)

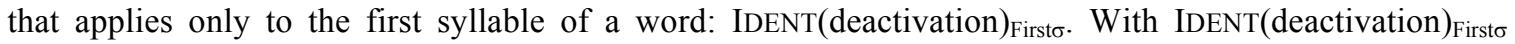
ranked above SELFDEACTIVATE, the contrast between self-deactivating and non-self-deactivating tongue root advancement gestures will be preserved in the first syllable of a word, and neutralized elsewhere.

The analysis of the distribution of ATR and RTR vowels in Classical Manchu is demonstrated by the following tableaux. The first tableau in (9) is for the word [sisə-ku] 'sieve,' in which an [i] in the initial syllable triggers harmony throughout the rest of the word. For reasons of space, only the vowel gestures for the input and output candidates are included, and the lip protrusion gestures responsible for the rounding of $/ \mathrm{u} /$ and $/ \mathrm{v} /$ are omitted.

Tableau for [sisə-ku] 'sieve'

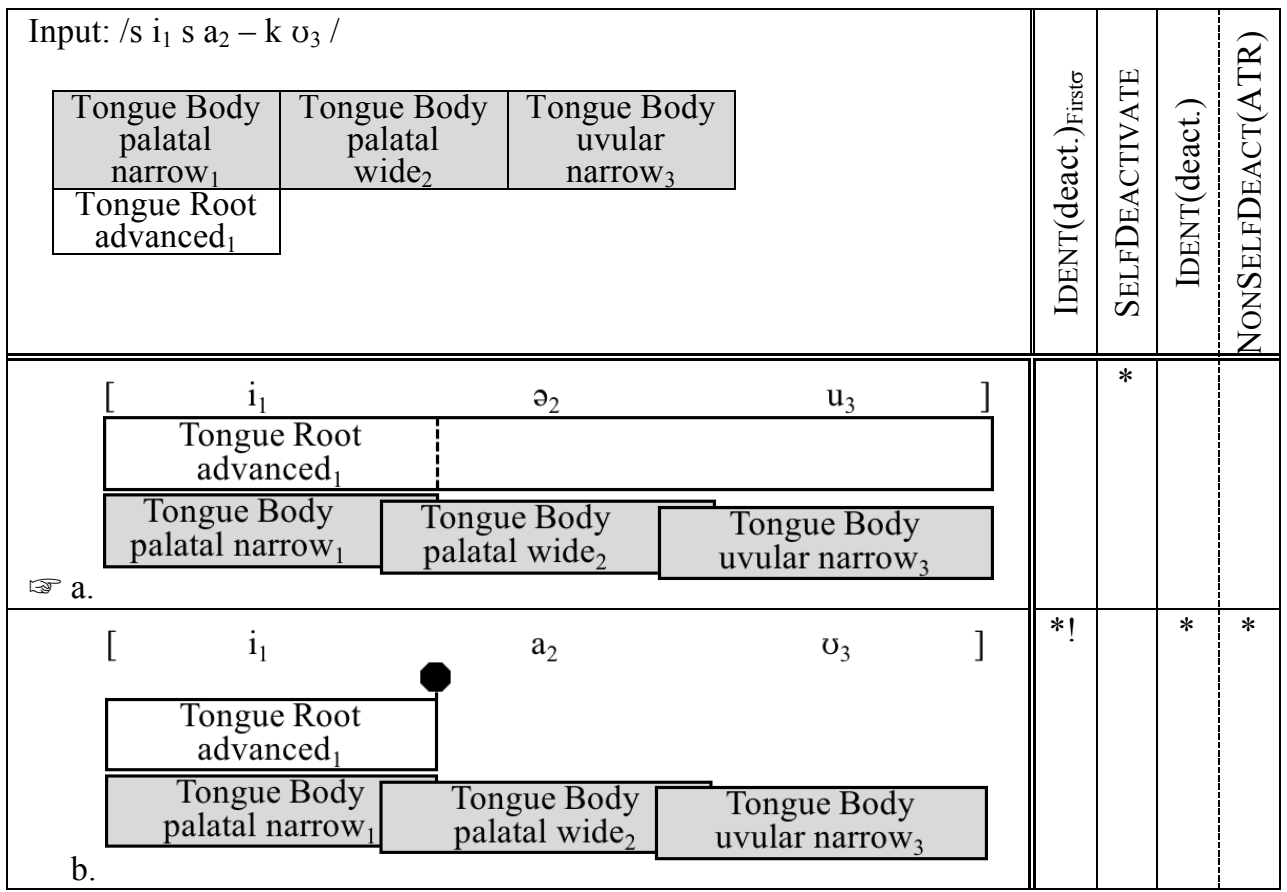


The input of the tableau in (9) contains the vowel /i/ accompanied by a non-self-deactivating tongue root advancement gesture. In both output candidates, this vowel is in the initial syllable. The winning candidate (a) [sisə-ku] surfaces with a faithfully realized non-self-deactivating tongue root advancement gesture, violating SELFDEACTIVATE but satisfying higher-ranked IDENT(deactivation) Firsto. In candidate (b) [sisa-ku] the tongue root advancement gesture is self-deactivating, satisfying SELFDEACTIVATE but fatally violating higher-ranked IDENT(deactivation) Firsto, as well as the low-ranked general IDENT(deactivation) and NONSElFDeActivate(ATR). The candidate that is faithful to the input's specified deactivation parameter for tongue root advancement is the winner.

The faithful candidate is also selected when the input contains a self-deactivating tongue root advancement gesture in an initial syllable. This is shown in the following tableau for [nilhひ-da] 'be slick.'

Tableau for [nilhø-da] 'be slick'

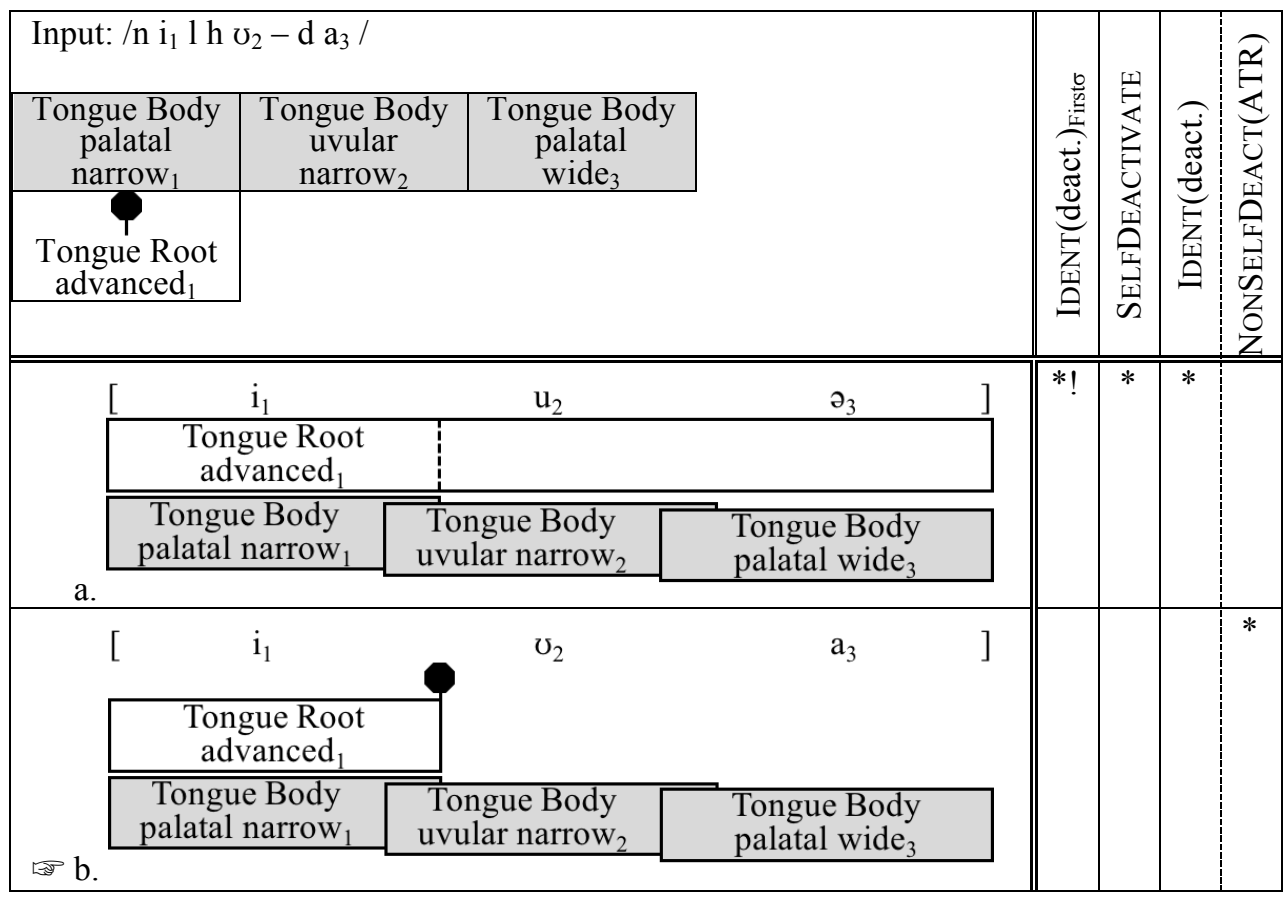

In (10), the input contains an /i/ accompanied by a self-deactivating tongue root advancement gesture. In candidate (a) [nilhu-də], this gesture surfaces as non-self-deactivating, violating both IDENT(deactivation) constraints as well as SELFDEACTIVATE. The winning candidate (b) [nilhひ-da] faithfully realizes the tongue root advancement gesture as self-deactivating, crucially satisfying high-ranked IDENT(deactivation) Firsto and violating only low-ranked NONSELFDeACTIVATE(ATR). This ranking has successfully preserved the contrast between self-deactivating and non-self-deactivating tongue root advancement gestures in an initial syllable.

However, when /i/ occurs in a non-initial syllable, it never triggers harmony, regardless of what its input specification for self-deactivating may be. This is demonstrated in the following tableau for [kani-nga] 'agreeing.' In order to demonstrate that a tongue root advancement gesture will always surface as self-deactivating in a non-initial syllable, an input with a non-self-deactivating tongue root advancement gesture is posited. 
Tableau for [kani-nga] 'agreeing'

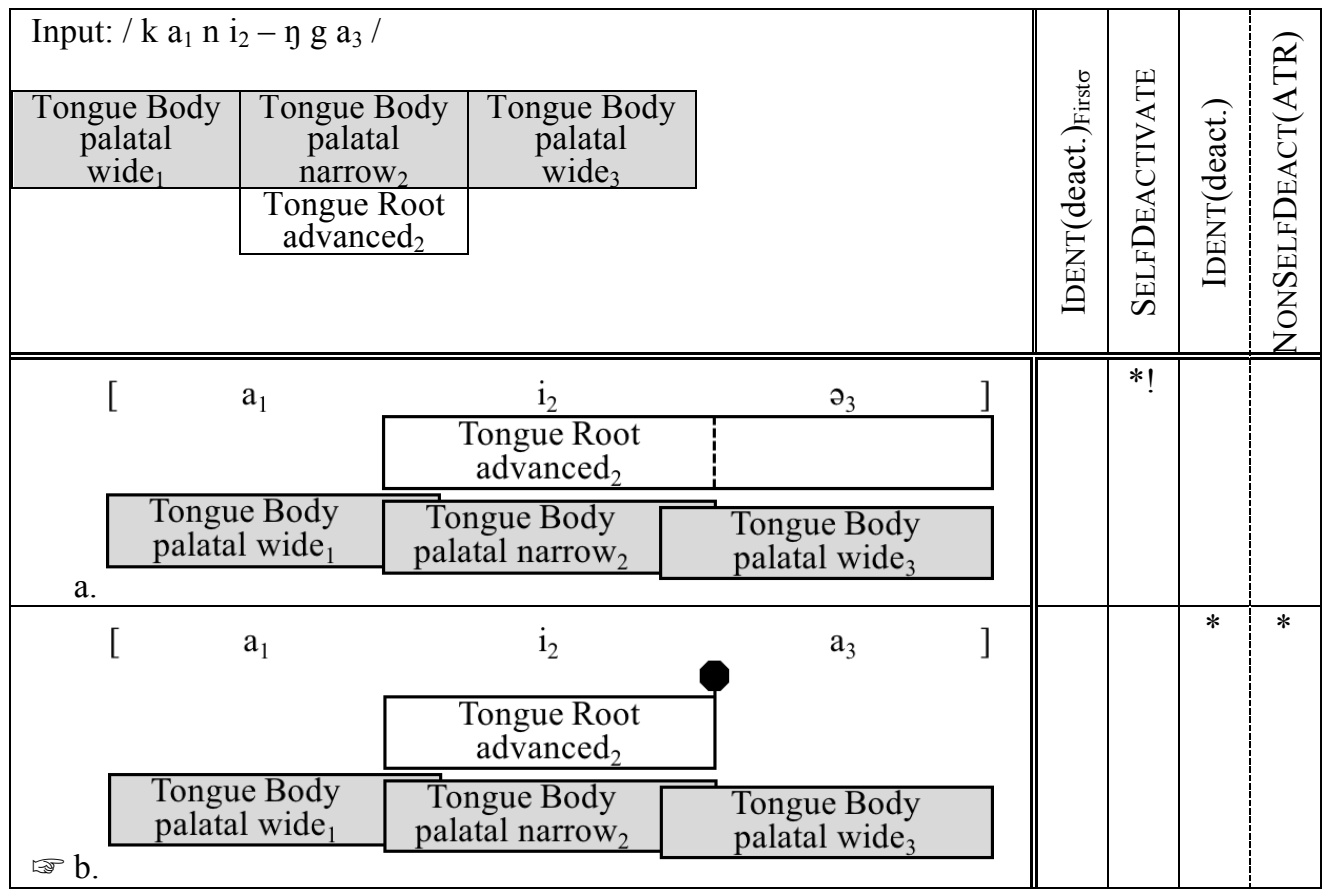

In (11), the undominated constraint IDENT(deactivation) Firsto is not relevant, as the tongue root advancement gesture of [i] does not occur in the initial syllable of either candidate output. Therefore, in this form the choice between candidates falls to the lower-ranked constraints. Candidate (a), [kani-nge], in which the tongue root advancement gesture of [i] is non-self-deactivating and therefore a trigger of harmony, fatally violates SelFDEActivate. Winning candidate (b) [kani-nga], on the other hand, satisfies this constraint with its self-deactivating tongue root advancement gesture at the expense of violating the low-ranked general IDENT(deactivation), as well as NONSELFDeActivate(ATR). This tableau shows that no matter a tongue root advancement gesture's input deactivating parameter setting, it will always surface as selfdeactivating in a non-initial syllable. This captures the fact that in Classical Manchu only vowels in the initial syllable of a word may trigger harmony.

The current ranking accounts for the fact that high ATR vowels exhibit contrastive triggering in Classical Manchu, and that this contrast is limited to the initial syllable. A final complication emerges from the fact that in an initial syllable the nonhigh ATR/a/ does not exhibit contrastive triggering but instead must trigger harmony exceptionlessly. It is necessary, then, to restrict/a/ from ever being accompanied by a self-deactivating tongue root advancement gesture. This can be achieved via a gestural co-occurrence constraint (Smith 2016c) that penalizes a nonhigh vowel that is accompanied by a self-deactivating tongue root advancement gesture. ${ }^{3}$ It is defined as follows:

*(Nonhigh Vowel, Self-deactivating ATR): Assign a violation mark to a nonhigh vowel gesture and a self-deactivating tongue root advancement gesture that are concurrently active.

This constraint must outrank IDENT(deactivation) Firsto $_{\text {; }}$ even in the privileged position of the initial syllable, triggering is not contrastive for /a/ in Classical Manchu. This ranking is summarized in (13).

\footnotetext{
${ }^{3}$ This type of co-occurrence constraint that references the deactivation parameter of a gesture can be used more generally to account for harmony systems in which conditions are imposed on the triggers of harmony. For more on the phonetic grounding of conditional triggering of harmony, see Kaun (1995), Walker (2005), and Kimper (2011).
} 
*(Nonhigh Vowel, Self-deactivating ATR)

(13)

\author{
$>$ \\ IDENT(deactivation) $)_{\text {Firsto }}$ \\ SELFDEACTIVATE \\ $>$ \\ IDENT(deactivation), NonSELFDEACTIVATE(ATR)
}

The ranking in (13) is demonstrated in the tableau in (14) for the form [hərə-ku] 'ladle.' In order to show that /o/ in an initial syllable will always surface with a non-self-deactivating tongue root advancement gesture and will therefore always trigger harmony, a hypothetical input containing a self-deactivating tongue root advancement gesture is posited.

Tableau for [hərə-ku] 'ladle'

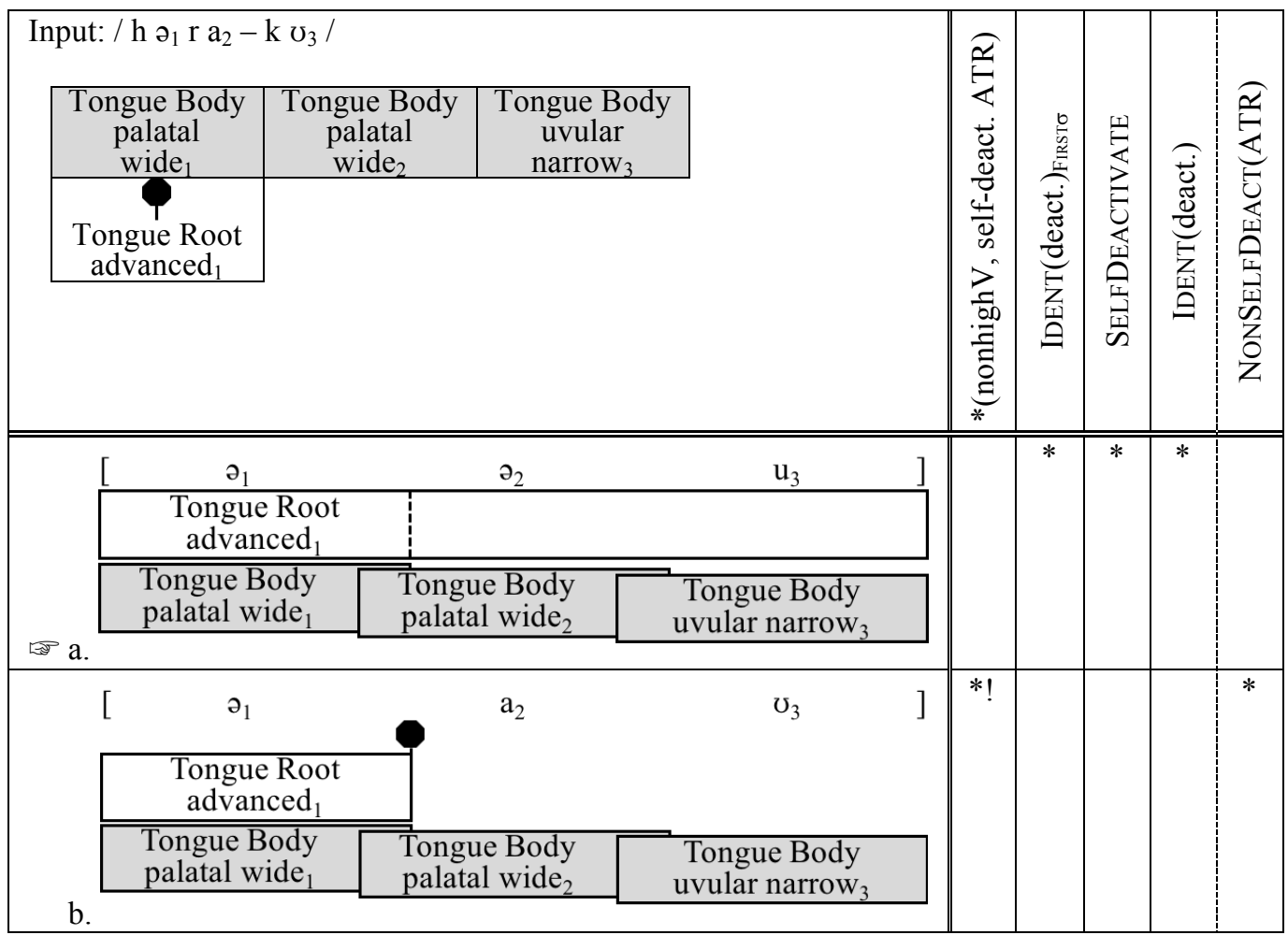

The tongue root advancement gesture in winning candidate (a) [hərə-ku] is non-self-deactivating and triggers ATR harmony, violating SELFDEACTIVATE as well as both IDENT(deactivation) constraints. The faithful candidate (b) [həra-kv] surfaces with a self-deactivating tongue root advancement gesture, satisfying all of these constraint but fatally violating highest-ranked *(Nonhigh Vowel, Self-deactivating ATR). This constraint requires a nonhigh ATR vowel to trigger harmony, regardless of the input specification of a tongue root advancement gesture. Both IDENT(deactivation) constraints are outranked and therefore are unable to preserve any underlying contrast in triggering ability among nonhigh ATR vowels.

Classical Manchu presents an exceptionally complex pattern of harmony triggering. High ATR vowels display contrastive triggering of tongue root harmony, while nonhigh ATR vowels are required to always trigger harmony. This is combined with the restriction of harmony triggers to the privileged position of the initial syllable. The Gestural Harmony Model successfully accounts for all of this through the interaction of constraints on a gesture's deactivation parameter, general and positional faithfulness constraints, and constraints on gestural co-occurrence. 
The complexity of this system of harmony triggering points to the importance of viewing the presence of harmony as the result of shaping of phonological inventories to include harmony-triggering gestures and of restricting those gestures to certain positions. A constraint that simply mandates harmony is unable to capture the full range of complexity shown by patterns of harmony triggering. Attempts to do so within feature-based analyses of harmony triggering are met with issues of both over- and under-generation of harmony triggering patterns. This is the subject of the next section.

\section{Alternative Account: Constraint Indexation}

The type of pattern observed in Classical Manchu ATR harmony is often thought of as a case of exceptionality in harmony triggering. As such, it must be analyzed using the types of mechanisms typical to analyses of phonological exceptionality. One of the most common mechanisms used for analyzing patterns of exceptionality in OT is morpheme indexation (Pater 2000, 2009). Exceptional harmony triggering can be achieved via indexation to a harmony driving constraint, such as the commonly used SPREAD(F) (Padgett 1995; Walker 1998/2000). However, such an approach both over- and under-generates patterns of harmony triggering, rendering it an undesirable alternative to the Gestural Harmony Model. The reasons for this are examined in this section.

The constraint SPREAD(F) drives unbounded harmony by penalizing non-undergoers, i.e., those segments that are not associated with a harmonizing feature F. It is defined as follows:

SPREAD(F): For each feature $\mathrm{F}$ associated to a segment in domain $\mathrm{D}$, assign a violation mark for every segment $\mathrm{S}$ in domain $\mathrm{D}$ that is not associated to F. (adapted from Walker $(1998$, p. 51))

When SPREAD(F) dominates IDENT(F), which preserves potential undergoers' underlying values for the feature $\mathrm{F}$, harmony results.

In order to account for the fact that in a language such as Classical Manchu only some forms show within- and across-morpheme harmony, constraint indexation can be used to relativize SPREAD(F) such that it does not apply to all forms. Instead, an indexed constraint will only be relevant when a word contains a morpheme that shares its index, and will only be violated if some portion of an indexed morpheme contributes to its violation. In harmony, patterns in which some forms display harmony while others do not can be analyzed by indexing triggering morpheme to a version of SPREAD(F) that outranks IDENT(F), which in turn outranks a general version of SPREAD(F). This ranking is shown in (16).

$$
\text { Constraint ranking for exceptional triggering of harmony }
$$

$$
\operatorname{SPREAD}(\mathrm{F})_{\mathrm{i}} \gg>\operatorname{IDENT}(\mathrm{F})>>\operatorname{SPREAD}(\mathrm{F})
$$

When the domain of SPREAD(F) is specified to refer to the entire word, the constraint compels both withinand cross-morpheme harmony. This is true of both the general SPREAD(F) constraint and the version that is indexed only to certain morphemes. Crucially, it is not the case that the entire structural description of an indexed constraint must be met only by material from an indexed morpheme. Rather, Pater (2009) specifies that an indexed constraint is violated if its structural description contains some part of an indexed morpheme.

Tableaux that demonstrate the workings of the ranking in (16) are provided in (17) and (18). Both include forms in which two segments $S_{1}$ and $S_{2}$ occupy a root while segment $S_{3}$ occupies a suffix attached to that root. In (17), the root morpheme is indexed to high-ranked SPREAD $(F)_{i}$. 
Tableau with harmony triggered by an indexed morpheme

\begin{tabular}{|c|c|c|c|c|}
\hline Input: $\left[\mathrm{S}_{1}\right.$ & $\left.\mathrm{S}_{2}\right]_{\mathrm{i}}\left[\mathrm{S}_{3}\right]$ & $\operatorname{SPREAD}(\mathrm{F})_{\mathrm{i}}$ & IDENT(F) & $\operatorname{SPREAD}(\mathrm{F})$ \\
\hline $\begin{array}{c}\mathrm{F} \\
\text { a. }\left[\mathrm{S}_{1}\right.\end{array}$ & $\left.\mathrm{S}_{2}\right]_{\mathrm{i}}\left[\mathrm{S}_{3}\right]$ & $* ! *$ & & $* *$ \\
\hline b. $\left[\mathrm{S}_{1}\right.$ & $\left.\mathrm{S}_{2}\right]_{\mathrm{i}}\left[\mathrm{S}_{3}\right]$ & & $* *$ & \\
\hline
\end{tabular}

In (17), the morpheme containing the potential trigger segment $S_{1}$, which is associated with feature $F$, is indexed to high-ranking $\operatorname{SpREAD}(\mathrm{F})_{\mathrm{i}}$. Output candidate (a) violates both the indexed and non-indexed versions of SPREAD(F) due to the lack of association between the harmonizing feature $F$ and segments $\mathrm{S}_{2}$ and $\mathrm{S}_{3}$. Winning candidate (b) satisfies both SPREAD constraints but violates IDENT(F) due to the added association lines between feature $F$ and undergoing segments $S_{2}$ and $S_{3}$.

The situation is different in the tableau in (18), in which the morpheme containing the potential trigger segment $\mathrm{S}_{1}$ bears no constraint indexation.

Tableau with harmony not triggered by an unindexed morpheme

\begin{tabular}{|c|c|c|c|c|}
\hline $\begin{array}{r}\mid \\
\text { Input: }\left[S_{1}\right. \\
\end{array}$ & $\left.\mathrm{S}_{2}\right]\left[\mathrm{S}_{3}\right]$ & $\operatorname{SPREAD}(\mathrm{F})_{\mathrm{i}}$ & $\operatorname{IDENT}(\mathrm{F})$ & $\operatorname{SPREAD}(\mathrm{F})$ \\
\hline$\stackrel{F}{\mid}$ & $\left.\mathrm{S}_{2}\right]\left[\mathrm{S}_{3}\right]$ & & & $* *$ \\
\hline b. $\left[\mathrm{S}_{1}\right.$ & $\left.\mathrm{S}_{2}\right]\left[\mathrm{S}_{3}\right]$ & & $* ! *$ & \\
\hline
\end{tabular}

In this tableau, winning candidate (a), which displays no harmony, violates only low-ranked $\operatorname{SPREAD}(\mathrm{F})$. This is because the morpheme containing the potential trigger segment $\mathrm{S}_{1}$ bears no index, and is therefore not subject to high-ranked $\operatorname{SPREAD}(\mathrm{F})_{\mathrm{i}}$. Harmonizing candidate (b) fatally violates IDENT(F) due to the added association lines between feature $F$ and segments $S_{2}$ and $S_{3}$. With this ranking, then, a system in which only morphemes indexed to a high-ranked version of SPREAD(F) may display harmony while others do not can be generated easily.

However, problems arise when considering that it is not only morphemes that contain the trigger of harmony that can be indexed to the constraint $\operatorname{SPREAD}(\mathrm{F})_{\mathrm{i}}$. Because the structural description of $\operatorname{SPREAD}(\mathrm{F})$ may contain segments from two different morphemes, provided the domain $\mathrm{D}$ of harmony is the prosodic word, there are two morphemes that potentially bear an indexation to the constraint. That is, either the trigger or the undergoer of harmony may be in an indexed morpheme that activates the indexed $\operatorname{SPREAD}(\mathrm{F})_{\mathrm{i}}$ constraint. This means that the SPREAD(F) constraint depicted in (19) is relevant when either segment $\mathrm{S}$ (the potential trigger or the potential undergoer) in the structural description is part of an indexed morpheme.

Structural description of SPREAD(F)

$$
\left.\begin{array}{lll}
* & F \\
\mid & & \\
S & \ldots & S
\end{array}\right\}_{D}
$$

A pathological pattern results when a morpheme containing a potential undergoer of harmony is indexed to $\operatorname{SPREAD}(\mathrm{F})_{\mathrm{i}}$ while a morpheme containing a potential trigger of harmony is not indexed. This is 
demonstrated in the tableau in (20), in which the triggering segment $\mathrm{S}_{1}$ bearing feature $\mathrm{F}$ is still in the root, but the indexation to $\operatorname{SPREAD}(\mathrm{F})_{\mathrm{i}}$ is now borne only by the affix containing $\mathrm{S}_{3}$. This situation results in harmony across the root, despite the fact that the root would surface as disharmonic if it did not have an indexed affix attached to it, as was the case in (18) above.

Tableau with harmony triggered across an unindexed morpheme due to indexed affix

\begin{tabular}{|c|c|c|c|c|}
\hline Input: $\left[\mathrm{S}_{1}^{\mathrm{F}}\right.$ & $\left.\mathrm{S}_{2}\right]\left[\mathrm{S}_{3}\right]_{\mathrm{i}}$ & SPREAD $(F)_{i}$ & IDENT(F) & SPREAD(F) \\
\hline $\begin{array}{c}\mathrm{F} \\
\mathrm{I} \\
\text { a. }\end{array}$ & $\left.S_{2}\right]\left[S_{3}\right]_{i}$ & $* !$ & & $* *$ \\
\hline b. $\left[\mathrm{S}_{1}\right.$ & $\left.\widehat{\left.S_{2}\right]\left[S_{3}\right.}\right]_{i}$ & & $* *$ & \\
\hline
\end{tabular}

In candidate (a) the lack of harmony for the feature $\mathrm{F}$ both within and across the morpheme boundary violates both versions of $\operatorname{SPREAD}(\mathrm{F})_{\mathrm{i}}$. Most crucially, the high-ranked indexed version of $\operatorname{SPREAD}(\mathrm{F})_{\mathrm{i}}$ is violated because the affix containing $S_{3}$ is indexed to it, and $S_{3}$ is not associated with feature $F$. The winning candidate (b) satisfies high-ranked $\operatorname{SPREAD}(\mathrm{F})_{\mathrm{i}}$ by spreading the feature $\mathrm{F}$ across the root and onto $\mathrm{S}_{3}$ in the indexed affix. In candidate (b), the segment $\mathrm{S}_{1}$ has triggered harmony in order to satisfy $\operatorname{SPREAD}(\mathrm{F})_{\mathrm{i}}$ despite the fact that $\mathrm{S}_{1}$ does not even occur in the morpheme bearing the indexation to that constraint. Therefore, while this hypothetical language usually has no within-morpheme harmony (due to the ranking of IDENT(F) over the general SPREAD(F) constraint, within-morpheme harmony has occurred in candidate (b) so that the feature F can spread to an indexed morpheme. Such a pattern is unattested according to Finley (2010), who claims that even in the presence of an affix that is an exceptional undergoer of harmony, harmony is never induced within an otherwise disharmonic stem. Morpheme indexation to a harmony-driving constraint has therefore generated an unattested, pathological pattern of harmony.

Furthermore, the constraint indexation approach is also incapable of generating certain aspects of the distribution of triggers and non-triggers of harmony. In Classical Manchu, the contrast between triggering and non-triggering segments that bear a harmonizing property is restricted to a privileged position, the initial syllable. This sort of preservation of phonological contrast only in privileged positions is common, and can be accounted for straightforwardly using positional faithfulness constraints (Beckman 1997, 1998) provided we assume that a segment's ability to trigger harmony is a potentially contrastive property. The Gestural Harmony Model, with its focus on analyzing harmony triggering patterns as the result of constraints on the positions in which self-deactivating and non-self-deactivating gestures may occur in a word, is well suited to accounting for such patterns. However, constraint indexation is unable to generate the different distributional patterns of triggering and non-triggering segments.

In a framework that assumes only morpheme indexation to harmony-driving constraints, this is unsurprising. A segment bearing feature $F$ that occurs in a morpheme indexed to $\operatorname{SPREAD}(\mathrm{F})_{\mathrm{i}}$ will trigger harmony no matter its position in the morpheme. The adoption of segmental rather than morphemic indexation, as proposed by Temkin Martínez (2010), does not seem to solve this problem. Markedness and faithfulness constraints, whether they are relativized to privileged positions or not, can reference properties of phonological units, such as feature values or gestural parameter settings. However, it is unclear whether indices to constraints are objects that can be referenced by constraints in this way. The index on a morpheme or segment carries no distinctive information on its own; it is only defined if there is some constraint in the grammar that shares that indexation. An index conveys information about the relation between a phonological unit and some element of the phonological grammar, not about the phonological unit itself. Thus, markedness and faithfulness constraints should not be able to make reference to any constraint indices a phonological unit might bear, and would therefore be of no use in determining the distribution of exceptional harmony triggers, i.e., segments bearing an index to $\operatorname{SPREAD}(\mathrm{F}) \mathrm{i}_{\mathrm{i}}$. 


\section{Conclusion}

The Gestural Harmony Model is successful in accounting for patterns in which a segment's ability to trigger harmony appears to be a contrastive property. Because gestural representations include a parameter for (non-)self-deactivation, this parameter can be referenced by markedness and faithfulness constraints that determine whether certain gestures are included in a language's phonological inventory, as well as their distributions. This paper demonstrates this with an analysis of the complex pattern of triggering of ATR harmony in Classical Manchu, which exhibits contrastive triggering by high vowels, obligatory triggering by nonhigh vowels, and distributional restrictions on triggers.

The Gestural Harmony Model presents a novel approach to accounting for patterns of harmony triggering that have previously fallen within the realm of phonological exceptionality. Analyses that rely on morpheme or segment indexation to harmony-driving constraints suffer from issues of both over- and under-generation of patterns of harmony triggering. The unique way in which the Gestural Harmony Model represents triggering segments makes it possible to account for complex and seemingly exception-laden patterns of harmony triggering without generating the pathological predictions of alternative feature-based analyses.

\section{References}

Beckman, Jill N. (1997). Positional faithfulness, positional neutralisation and Shona vowel harmony. Phonology, 14, 146.

Beckman, Jill N. (1998). Positional Faithfulness. Ph.D. Dissertation, University of Massachusetts Amherst.

Browman, Catherine P., \& Goldstein, Louis. (1986). Towards an Articulatory Phonology. Phonology Yearbook, 3, 219-252.

Browman, Catherine P., \& Goldstein, Louis. (1989). Articulatory gestures as phonological units. Phonology, 6(2), 201251.

Finley, Sara. (2010). Exceptions in vowel harmony are local. Lingua, 120(6), 1549-1566.

Kaun, Abigail. (1995). The Typology of Rounding Harmony: An Optimality Theoretic Approach. Ph.D. Dissertation, University of California Los Angeles.

Kimper, Wendell. (2011). Competing Triggers: Transparency and Opacity in Vowel Harmony. Ph.D. Dissertation, University of Massachusetts Amherst.

Padgett, Jaye. (1995). Partial Class Behavior and Nasal Place Assimilation. In K. Suzuki \& D. Elzinga (Eds.), Proceedings of the Arizona Phonology Conference: Workshop on Features in Optimality Theory (pp. 145-183). Tucson: University of Arizona.

Pater, Joe. (2000). Non-uniformity in English secondary stress: the role of ranked and lexically specific constraints. Phonology, 17, 237-274.

Pater, Joe. (2009). Morpheme-Specific Phonology: Constraint Indexation and Inconsistency Resolution. In S. Parker (Ed.), Phonological Augmentation: Essays on Evidence and Motivation (pp. 123-154). London: Equinox.

Smith, Caitlin. (2016a). Nasal Spreading as Defective Gestural Deactivation. In Supplemental Proceedings of the 2014 Annual Meeting on Phonology.

Smith, Caitlin. (2016b). A gestural account of neutral segment asymmetries in harmony. In Proceedings of the 2015 Annual Meeting on Phonology.

Smith, Caitlin. (2016c). Morphological Consonant Mutation as Gestural Affixation. In Proceedings of the Fiftieth Annual Meeting of the Chicago Linguistic Society (pp. 411-426).

Temkin Martínez, Michal. (2010). Sources of Non-Conformity in Phonology: Variation and Exceptionality in Modern Hebrew Spirantization. Ph.D. Dissertation, University of Southern California.

Vago, Robert M. (1973). Abstract Vowel Harmony Systems in Uralic and Altaic Languages. Language, 49(3), 579605.

Walker, Rachel. (2001). Round Licensing, Harmony, and Bisyllabic Triggers in Altaic. Natural Language \& Linguistic Theory, 19, 827-878.

Walker, Rachel. (2005). Weak Triggers in Vowel Harmony. Natural Language \& Linguistic Theory, 23(4), 917-989.

Walker, Rachel. (1998). Nasalization, Neutral Segments, and Opacity Effects. Ph.D. Dissertation, University of California Santa Cruz. Published 2000 by Garland Publishing.

Zhang, Xi. (1996). Vowel Systems of the Manchu-Tungus Languages of China. Ph.D. Dissertation, University of Toronto.

Zhang, Xi, \& Dresher, B. Elan. (1996). Labial Harmony in Written Manchu. Saksaha: A Review of Manchu Studies, 1, $13-27$. 